\title{
Approaches to Improve Clinical Efficacy of CD19- Redirected Chimeric Antigen Receptor (CD19 CAR) T Cell Immunotherapy of Non-Hodgkin's Lymphoma
}

\author{
*Ali R Jazirehi and Tam NM Dinh \\ Department of Surgery, Division of Surgical Oncology, University of California at Los Angeles, USA
}

Submission: June 23, 2017; Published: July 21, 2017

"Correspondence Address: Ali Jazirehi, CLS, Division of Surgical Oncology, University of California, Los Angeles, CHS 54-140, 10833 Le Conte Avenue, Mail code: 178218, Los Angeles, CA 90095, USA, Tel: (310) 206-8509; Fax: (310) 267-2679; Email: ajazirehi@mednet.ucla.edu

\begin{abstract}
Clinical use of CD19-redirected chimeric antigen-receptor (CD19 CAR) T cell therapy has shown promise in the treatment of various B cell malignancies including acute lymphoblastic leukemia (ALL) and non-Hodgkin's lymphoma (NHL). However, its clinical use is limited due to inherent or acquired resistance of tumor cells to apoptotic death signals delivered by CAR transduced T cells as well as by the undesired side effects. Approaches to improve the clinical efficacy of CAR T cell therapy is urgently needed. In 2006, the histone deacetylase inhibitor vorinostat (SAHA) received Food and Drug Administration (FDA) approval for treatment of cutaneous T-cell lymphoma. It affects the acetylation of histones and centromeres, and non-histone proteins, and regulates gene expression. Similarly, the nonsteroidal anti-inflammatory drug (NSAID) Celecoxib (Celebrex®) received FDA approval in 1998 for treatment of osteoarthritis and rheumatoid arthritis; its use is extended to other cancers.
\end{abstract}

NHL is highly dependent on inflammatory micro-environment for its growth, which is inhibited by celecoxib. Celecoxib and HAD Cihinder tumor growth via regulation of various apoptotic genes, such as cyclooxygenase-2 (Cox-2), pro- and anti-apoptotic Bcl-2 family members, Akt/PKB pathway, IAP family members, and other cell survival signaling pathways. Moreover, technological advances to incorporate various suicide gene systems in eukaryotic cells has limited the non-selective effects and reduced serious adverse effects (SAEs) of CD19 CAR T cell therapy. Considering their broad regulatory effects on apoptotic gene products, Celecoxib and HDACiare promising drugs to improve CD19CAR T cell therapy.CD19 CAR T cell immunotherapy of NHL can further be optimized through incorporation of suicide gene systems.

Keywords: CD19; Chimeric antigen receptor; Antibody mediated treatment; Immunotherapy; Suicide gene; Celebrex; Histone deacetylase inhibitor; Signal transduction; Apoptosis; Non-Hodgkin's lymphoma; SAHA; Panobinostat

\section{Chimeric Antigen Receptors- Novel Immunotherapy for Non-Hodgkin's Lymphoma (NHL)}

Engineering $\mathrm{T}$ cells to directly target tumor associated antigens (TAA) helps eliminate the requirement for MHCmediated peptide presentation as most tumors down-regulate surface MHC in an attempt to avoid immune detection and destruction Cheadle et al. [1]. Chimeric antigen receptor transduced human peripheral blood T lymphocytes (CAR T cells) meet this criterion. A CAR T cell commonly has an antigen-binding domain, which is encoded by single chain variable fragments (scFv), CD28 co-stimulatory domain, and $\mathrm{CD} 3 \zeta$ signaling domain that triggers $\mathrm{T}$ cell activation Davila et al. [2], Kenderian et al. [3]. For CD19 CAR T cells, scFv is specific for the CD19 receptor, directing the CAR T cells selectively to CD19+ NHL cells Davila et al. [4]. CD19 CAR therapy has shown potential as an alternative cancer therapy for B cell malignancies. In a phase I/II trial, fortyone patients with NHL were infused with CD19 CAR T cells containing a defined ratio of CD8+ and CD4+ CAR T; an objective response rate (ORR) of $84 \%$ and a complete response rate (CR) of $47 \%$ were observed in those who received CD19 CAR T cells in addition to cyclophosphamide and fludarabine Turtle et al. [5]. Phase I trial involving patients with chemo-refractory NHLs underwent CAR $\mathrm{T}$ cell therapy following autologous hematopoietic stem cell transplantation (HSCT) showed modest results Wang et al. [6]. In NHL1 trial, out of eight patients who received HSCT and CD19 CAR T cells infusion, 3 (38\%) had a CR and $2(25 \%)$ had a partial response (PR); in NHL2 trial, out of 8 patients, $6(75 \%)$ had a CR and 2 (25\%) had a PR Wang et al. [6]. 


\section{Cancer Therapy \& Oncology International Journal}

\section{Potential Mechanisms of CD19 CAR T cell-induced Apoptosis-Resistance in NHL}

The modest to low response rates observed in clinical trials might be due to resistance mechanisms that NHLs have developed to avoid apoptosis induced by CD19 CAR T cells. CD19 antigen expression on tumor cells might become lost or down regulated after infusion of CD19 CAR T cells. Frame shift mutations that result in deletion of $\mathrm{C}$-terminal tyrosine residues critical for signal transduction through CD19, were reported in patients with very low or undetectable levels of surface CD19 Van Zelm et al. [7]. A patient who had normal CD19 allele but have defect in CD81 gene was reported to also lack CD19 expression Van Zelm et al. [8]. Since CD81 works in conjunction with CD19/ CD21 complexes in B cell receptor (BCR) signaling, it is possible that defects in CD81 will affect the expression of CD19 Cherukuri et al. [9]. Thus, CD81 defective individuals are non-responsive to CD19-redirected CAR therapy.

NHLs could potentially undergo alternative differentiation and signaling pathways to avoid recognition by CD19 CAR T cells. The biopsy of a patient with plasmablastic lymphoma (PBL) contained tumor cells lacking CD19 and other markers of pre-plasmacytic B-cell differentiation, suggesting that PBL might have used alternative B-cell differentiation pathways to proliferate and avoid apoptosis Evans et al. [10]. Mutations in genes that code for key regulators in cell proliferation and survival signaling pathways might also play a role in apoptosis resistance in tumor cells. Mutated BRAF, NRAS, and p53 genes, all of which are involved in cell cycle regulation, have been reported in a wide range of cancers Davies et al. [11], Daya-Grosjean et al. [12], Chiappetta et al. [13]. Aberrant BCR signaling pathways, including those that involve the Src family kinases Lyn, Syk, PI3K/Akt/mTOR, Bruton tyrosine kinase (Btk), NF- $\kappa B$, have been observed in chronic lymphocytic leukemia (CLL) and NonHodgkin's lymphoma (NHL) Woyach et al. [14], Arita et al. [15]. Abnormal levels of pro- and anti-apoptotic proteins expressed in tumor cells might also be responsible for apoptosis resistance. Whether the extrinsic and intrinsic apoptotic pathways are initiated or shut down depends on the balance between the expression levels of pro- and anti-apoptotic proteins A common trend found among various types of cancers is a decrease in Bax and Bak (pro-apoptotic members of Bcl-2 family) levels and an increase in Bcl-2, Mcl-1, and Bfl-1 (anti-apoptotic members of Bcl-2 family) Bentires-Alj et al. [16], Placzek et al. [17].

Other factors that might cause apoptosis resistance include physical barriers and an immunosuppressive tumor microenvironment. Adhesion of T-cells to tumor cells requires receptors/ligands recognition. Tumor cells might lack receptors such as intercellular adhesion molecule (ICAM)-1, P-selectin, and vascular cell-adhesion molecule (VCAM)-1 Agace [18]. CD40/ CD40 ligand (CD40L) interaction, which promotes E-selectin dependent leukocyte attachment, might also be disrupted in tumor cells Urban et al. [19]. Tumor cells are capable of inhibiting
T-cells proliferation by releasing immunosuppressive cytokines, such as transforming growth factor (TGF)- $\beta$ and interleukin (IL)10; these cytokines, in turn, indirectly drive differentiation of regulatory T-cells (Tregs), which suppress T-cell activities, and M2 macrophages. M2 macrophages are pro-tumorigenic and capable of producing more TGF- $\beta$ and IL-10 Onea \& Jazirehi [20], Enblad et al. [21]. In 2014, 3 patients with acute lymphocytic leukemia (ALL) had relapsed after early loss of CD19 CAR T-cells from the peripheral blood at 2 weeks to 3 months. The relapsed ALL cells, however, remained CD19-positive Maude et al. [22].

CAR T cell therapy can result in long-term toxicities because the CAR transduced T-cells can persist in the circulation even after cessation of treatment. One of these effects is the release of large quantities of cytokines from activated CAR $\mathrm{T}$ cells. Elevated cytokine levels can cause cytokine release syndrome or cytokine storm in more severe cases. During cytokine storm, the immune system creates a positive feedback loop of proinflammatory cytokine production. Cytokine storm is associated with hypotension and systematic inflammatory reaction syndrome where the entire body develops severe inflammation. These immune reactions are typically resolved using steroids or neutralizing antibodies, however, they can be fatal in some cases. Neurological toxic effects such as delirium, aphasia, hallucinations, and seizure-like activity have also been reported upon CAR T cell therapy Onea \& Jazirehi [20].

Several strategies have been proposed to overcome the resistance mechanisms of NHL cells to CAR T cell therapy and to reduce the serious adverse effects (SAE). These approaches include the use of FDA-approved drugs with proven anti-cancer properties, such as histone deacetylase inhibitors (HDACi) and celecoxib, in combination with CD19 CAR T cell therapy. Incorporation of various suicide gene systems will further optimize and improve the clinical efficacy of CAR T cell therapy.

\section{Use of Histone Deacetylase Inhibitors (HDACi) to Improve CAR T Cell Immunotherapy}

Histone deacetylase inhibitors (HDACis) are promising anti-tumor agents. In eukaryotic cells, histone acetylation and deacetylation processes are central to regulation of transcription and gene expression $\mathrm{Xu}$ et al. [23]. The balance between these two processes is required for normal cell growth; improper activity of histone acetyltransferases (HAT) and deacetylases (HDAC) are involved in the development of several diseases including cancer Lehrmann et al. [24]. Histone deacetylase inhibitors (HDACi) specifically target histone deacetylases, and alter gene transcription selectively Gui et al. [25]. Depending on their sequence homology and expression patterns, HDACs are generally divided into four classes: Class I, II, and IV are zincdependent deacetylases, while class III are NAD+-dependent deacetylases Smith et al. [26]. Hydroxamic acid-based vorinostat, also known as suberoylanilide hydroxamic acid (SAHA) and ZolinzaTM, inhibits class I, II, and IV deacetylases Xu et al. [23]. 
SAHA does not act on class III deacetylases, but sirtinol, a SIRT inhibitor, is capable of inhibiting class III deacetylases Wang et al. [27]. The ability of HDAC is to regulate the expression of apoptotic genes makes them effective anti-tumor agents.

Tumor necrosis factor-related apoptosis-inducing ligand (TRAIL) induces apoptosis in tumor cells, yet leaving untransformed cells mostly unaffected Corazza et al. [28]. HDAC is have been shown to up regulate TRAIL death receptor-5 (DR-5), and the combined use of HDACi and TRAIL induced Bid cleavage and activated caspase cascade $(-3,-8,-9,-10)$, all of which are involved in extrinsic apoptotic pathways Nakata et al. [29]. HDACi also induce apoptosis via the intrinsic apoptosis pathways. HDACi reduce the expression of key antiapoptotic proteins, such as Bcl-xL and myeloid cell leukemia-1 (Mcl-1) of the Bcl-2 proteins family and X-linked inhibitor of apoptosis (XIAP), while increase the expression of proapoptotic proteins Bim, Bax, and Bak of the Bcl-2 family Zhang et al. [30]. This will create a proapoptotic milieu and primes the cells to undergo apoptosis.

Another potent HDACi capable of inhibiting class I, II, and IV HDACs is panobinostat, also known as LBH589 Atadja [31]. LBH589 interferes with several processes involved in cell cycle regulation and apoptosis, thus, hindering tumor angiogenesis. It has several effects on endothelial cells, including inhibiting extracellular signal-regulated kinase 1/2 (ERK1/2) phosphorylation and expression of chemokine receptor CXCR4 Qian et al. [32]. In UACC62 melanoma cell line, short-term exposure to LBH589 significantly decreased the expression of microphthalmia-associated transcription factor (MITF) Yokoyama et al. [33]. Its interaction with p21, a negative regulator of p53-dependent and p53-independent apoptosis, and Bcl-2 family proteins makes MITF an important regulator of apoptosis Gartel et al. [34], Levy et al. [35]. LBH589 can indirectly induce apoptosis of tumor cells via MITF. By down regulating phosphorylated signal transducer and activator of transcription (STAT)-3 and STAT-5, LBH589 could also induce cytotoxicity of cutaneous T-cell lymphoma (CTCL) cells that had developed resistance to SAHA Shao et al. [36]. Considering their pleotropic effects, HDACis are suitable candidates for incorporation in clinical CAR T cell therapy protocols.

\section{Use of Celebrex (Celecoxib) to Improve CAR T Cell Immunotherapy}

Celecoxib, also known as Celebrex, is a nonsteroidal antiinflammatory drug (NSAID) commonly used to treat rheumatic diseases. Due to its ability to inhibit cyclooxygenase-2 (Cox-2), in recent years Celecoxib has been used to treat several types of malignancies, including breast, colorectal, and prostate cancers, Gupta et al. [37] Arun et al. [38], Hsu et al. [39]. Cox-2 facilitates tumor growth via its enzymatic product prostaglandin E2 (PGE2) Greenhough et al. [40]. PGE2 interacts with several other proteins to create an environment favorable for tumor development. It induces the expression of angiogenesis regulators interleukin- 6
(IL-6) and haptoglobin, and up regulates the expression of Mcl1 via PI3K/Akt-dependent pathway Gallouet et al. [41], Lin et al. [42]. By inhibiting Cox-2, celecoxib can indirectly inhibit the expression of PGE2, thus, reducing tumor growth.

Furthermore, celecoxib down regulates Mcl-1 expression, allowing proapoptotic Bcl-2 family members Bax and Bak to trigger cytochrome c release and to initiate intrinsic apoptotic pathway Wei et al. [43], Rudner et al. [44]. In Cox-2 deficient gastric cancer cells, celecoxib induced caspases-dependent apoptosis via the Akt/GSK3 $\beta / N A G-1$ pathway, inhibited phosphorylation of Akt (a protein involved in cell proliferation), and GSK3 $\beta$ (downstream target of Akt), and up regulated the expression of NAG-1 (a pro-apoptotic and anti-tumorigenic protein) Pang et al. [45]. Celecoxib was also down regulated the expression of survivin, a multifunctional member of the IAP family that interferes with caspases activation Jendrossek [46]. The regulatory effects of Celecoxib on apoptotic gene expression profile as well as resolving inflammation make Celecoxib and ideal drug to be used in combination with CAR T cell therapy to circumvent resistance.

\section{Incorporation of Suicide Genes to Improve CAR T Cell Immunotherapy}

Clinical utilization of CAR T cell based immunotherapy has shown promising results in various tumor models, however, its clinical efficacy is somewhat hampered, in part, due to cytokine release syndrome (CRS) as well as on target/off tumor effects Minagawa et al. [47], Zhang \& Xu [48]. Moreover, due to wide distribution pattern of tumor associated antigens (TAAs) on tumor and healthy cells, CAR $\mathrm{T}$ cells can potentially target healthy cells, causing serious adverse events (SAEs) Casucci \& Bondanza [49]. Various novel approaches have been designed to limit the non-selective effects of CAR T cells. These approaches include incorporation of suicide gene(s), inhibitory CAR, and the addition of exogenous molecules as a safety mechanism to control the activity of CAR T cells Zhang \& Xu [48].

\section{Incorporation of Suicide Gene:}

In this approach, a genetically encoded molecule capable of inducing apoptosis is used for selective destruction of adoptively transferred CAR $\mathrm{T}$ cells Jones et al. [50]. The ideal suicide gene should ensure irreversible selective elimination cells responsible for unwanted toxicity. Additionally, an ideal suicide gene should have adequate bio-availability and bio-distribution profiles Jones et al. [50], Minagawa et al. [47]. Currently, several suicide gene technologies have become available. Based on their mechanisms of action, these technologies can be classified into three categories: metabolic, dimerization inducing, and therapeutic monoclonal antibody-mediated (mAb).

\section{Gene-Directed Enzyme Prodrug Therapy (GDEPT)}

Using metabolic machineries, in genetically engineered CAR T cells a nontoxic drug is converted to a toxic compound Springer \& Niculescu-Duvaz [51]. A prime example of this approach is the 
use of Herpes simplex thymidine kinase (HSV-TK)/ganciclovir (GCV) suicide system Bonini et al. [52], where the nucleotide analogs acyclovir and GCV are phosphorylated by HSV-TK. DNA polymerase will incorporate the phosphorylated byproducts into growing DNA chain, resulting in chain termination and apoptosis Moolten [53] eliminating alloreactive $\mathrm{T}$ cells in allogeneic settings. However, as the origin of HSV-TK is viral, the HSV-TK/GCV system can potentially trigger the immune system to reject genetically Engineered CAR transduced T cells Casucci \& Bondanza [49], Minagawa et al. [47], Ciceri et al. [54], Bonini et al. [52], Tiberghien et al. [55], thus, limiting the use of this approach.

\section{Incorporation of Inducible Caspase-9 (iCasp9) to Improve CAR T Cell Immunotherapy}

With the advance of genome-editing technology, the dimerization inducing mechanism of suicide gene using inducible caspase 9 (iCasp9) has become more efficacious. iCasp9 gene contains the intracellular portion of human caspase-9 fused to a drug-binding domain derived from human FK506 protein Clackson et al. [56]. The Clustered Regularly Interspaced Short Palindromic Repeats (CRISPR)/Cas9 genome editing technology allows large-scale production of CD19redirected CAR $\mathrm{T}$ cells that are directed to $\mathrm{T}$ cell receptor (TCR) $\alpha$ constant (TRAC) locus in mouse model of acute lymphoblastic lymphoma (ALL), resulting in uniform CAR T cell expression in human peripheral blood $\mathrm{T}$ cells and augmentation of $\mathrm{T}$ cell proliferation and cytotoxic potential Eyquem et al. [57]. Genome editing technologies also allows for the synthesis of iCasp9, which being used in combination with the chemical induction of dimerization (CID) drug AP1903 Gargett \& Brown [58]. Upon intravenous administration, AP1903 induces the cross-linking of the drug-binding domain of the fused protein to dimerize and auto catalytically cleave caspase- 9 and activate executioner caspase- 3, leading to apoptosis Gargett \& Brown [58]. In contrast to HSV-TK/GCV suicide system, iCasp9 is non-immunogenic and allows for rapid elimination of CAR T cells; however, the efficacy of iCasp9 system needs to be optimized Jones et al. [50].

\section{Use of FDA Approved Therapeutic mAb to Improve CAR T Cell Immunotherapy}

In addition to iCasp9, approaches using therapeutic mAbmediated mechanism can be utilized to circumvent immunogenic responses and improve CAR $\mathrm{T}$ cell therapy. The chimeric mouse anti-human CD20 mAb (Rituximab, Rituxan $囚$ ) is most commonly used. In this approach, CAR T cells are engineered to express CD2 0 receptors, and after adoptive transfer of CAR T cells, anti-CD20 mAb is administered resulting in the elimination of unwanted or excess CAR T cells Introna et al. [59], Serafini et al. [60], Griffioen et al. [61]. However, CD20 receptors are also expressed by healthy B cells, so this approach can cause ontarget toxicity and hamper humoral immunity Minagawa et al. [47].
In addition to CD20 receptors, CAR T cells can be designed to express or not express certain receptors to enhance their potency. CAR $\mathrm{T}$ cells therapy has been reported to be unsuccessful partly due to tumor-induced immunosuppressive mechanisms, namely the production of adenosine Beavis et al. [62]. In tumor microenvironment, adenosine is being produced at immunosuppressive concentrations Ohta et al. [63]. Targeting the adenosine receptor A2AR (using A2AR antagonists or short hairpin RNA [shRNA]) in a murine model of HER2+ selfantigen tumors greatly improved the efficacy of CAR T cell-based immunotherapy. This effect was more pronounced when CART cell therapy was combined with other checkpoint inhibitors such as mAbs directed against program death (PD)-1 Beavis et al. [62].

\section{Conclusion}

Recent advances in using the immune system to treat NHL suggesta promising role of CD19 CAR T cell therapy in NHL clinical therapy. However, a subset of NHLs is either inherently resistant or develop resistance to CAR T cell-mediated immunotherapy upon long-term exposure to genetically engineered CAR T cells. Outgrowth of apoptosis-resistant malignant B cells is a major hurdle in successful NHL clinical therapy. Aberrant activity of signaling pathways and distorted apoptotic machinery concludes in NHLs that develop cross-resistance to anti-cancer agents with different modes of action. Thus, it is practical to develop new means to regulate apoptotic machinery to circumvent resistance. Based on the broad apoptotic gene regulatory effects of Celecoxib and HDACi, we propose that combination of CD19CAR T cell therapy and these FDA approved anti-cancer drugs can potentially improve the outcome of CD19CAR T cellbased treatment of NHL patients.

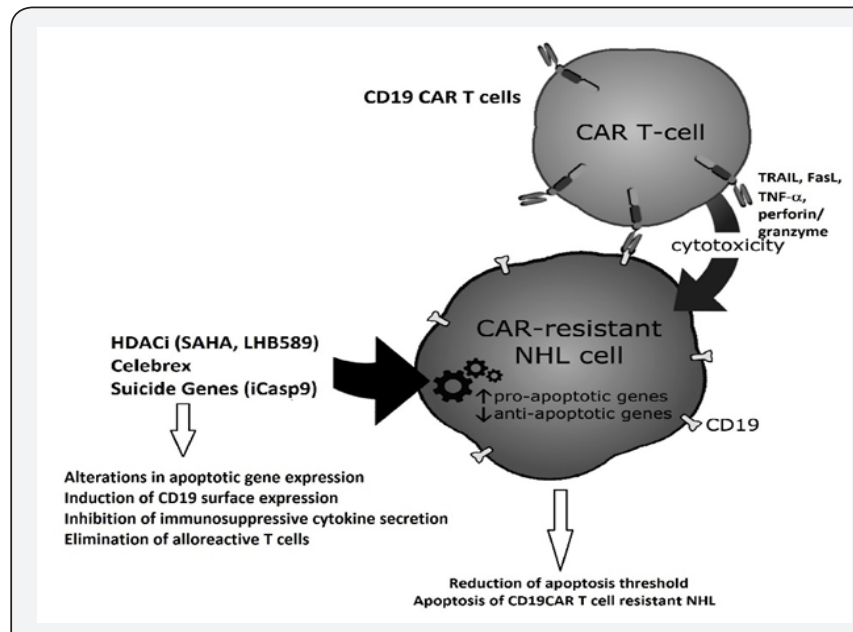

Figure 1: Improving CD19-redirected CAR T cell therapy of NHL.

Another challenge with CAR T cells therapy is that genetically modified $\mathrm{T}$ cells can persist in patients long after infusion. These $\mathrm{T}$ cells can induce various AEs including delirium, aphasia, hallucinations, seizure-like activity, fever, hypotension, and 


\section{Cancer Therapy \& Oncology International Journal}

hypoxia. Alloreactive CD19 CAR T cells can be eliminated via incorporation of various suicide gene systems into genetically modified T cells, thus, reducing the undesired toxic side effects. Future studies are warranted to optimize the schedule and doses of CD19 CAR T cell therapy (alone or combined with other drugs) to evade development of NHL resistance to CD19 CAR T cells, high grade AEs, and progressive immunodeficiency (Figure 1).

CD19-redirected CAR T cells can induce apoptosis in CD19+ sensitive NHL B-cells via TRAIL (Apo2L), FasL, TNF- $\alpha$, and perforin/granzyme apoptotic pathways. However, prolonged exposure of NHL B-cells to CD19 CAR T cells results in selective outgrowth and expansion of resistant cells. Resistant NHL cells have distorted apoptotic machinery and exhibit resistance to CD19CAR T cells despite adequate surface CD19 expression. The anti-inflammatory drug Celebrex ${ }^{\circledR}$ (Celecoxib), and the chromatin remodeling drug HDACi(SAHA and panobinostat[LBH 589]) regulate the expression profile of apoptotic genes, favoring the generation of a pro-apoptotic milieu; thus, CD19 CAR T cell-resistant NHL cells undergo apoptosis upon receiving death signals from CD19CAR T cells. Incorporation of suicide gene system in clinical CD19CAR T cell protocols will eliminate alloreactive CAR T cells, hence, reducing the undesired toxic side effects.

\section{Conflict of Interest}

The authors claim no conflicts of interest.

\section{References}

1. Klaus B, Matthias G, Gerd M (2007) A comparison between 2-Step IMRT and conventional IMRT planning. Radiotherapy and Oncology 84(3): 298-306.

2. Graham MV, Purdy JA, Emami B (1995) Preliminary results of a prospective trial using three dimensional radiotherapy for lung Cancer. Int J Rad Oncol Biol Phys 33: 993-1000.

3. Marks LB, Munley MT, Bentel GC (1997) Physical and biological predictors of changes in whole-lung function following thoracic irradiation. Int J Rad Oncol Biol Phys 39: 563-570.
4. Martel MK, Ten Haken RK, Hazuka M (1994) B. Dose-volume histogram and 3-D treatment planning evaluation of patients with pneumonitis. Int J Rad Oncol Biol Phys 28: 575-581.

5. Patz EF, Lowe VJ, Goodman PC, Herndon J (1995) Thoracic nodal staging with PET imaging with 18FDG in patients with bronchogenic carcinoma. Chest 108: 1617-1621.

6. Nestle U, Walter K, Schmidt S, Sybrecht GW, Schnabel K, et al. (1999) 18F-deoxyglucose positron emission tomography (FDG PET) for the planning of radiotherapy in lung cancer: High impact in patients with atelectasis. Int J Radiat Oncol Biol Phys 44: 593-59.

7. Sasaki M, lchiya Y, Kuwabara Y, et al. (1996) The usefulness of FDG positron emission tomography for the detection of mediastinal lymph node metastases in patients with non-small cell lung cancer: a L.J Vanuytsel et al. / Radiotherapy and Oncology 55 (2000) 317 \pm 324323 comparative study with X-ray computed tomography. Eur J Nucl Med 23: 741-747.

8. Valk PE, Pounds TR, Hopkins DM, et al. (1995) Staging non-small cell lung cancer by whole-body positron emission tomographic imaging. Ann Thorac Surg 60(6): 1573-1582.

9. Wahl RL, Quint LE, Greenough RL, White RI, Orringer MB, et al. (1994) Staging of mediastinal non-small cell lung cancer with FDG PET. CT and fusion images: Preliminary prospective evaluation. Radiology 191: 371-377.

10. Chin R, Ward R, Keyes JW (1995) Mediastinal staging of non-small cell lung cancer with positron emission tomography. Am J Respir Crit Care Med 152: 2090-2096

11. Vansteenkiste JF (1998) FDG-PET scan in potentially operable nonsmall cell lung cancer: do anatometabolic PET-CT fusion images improve the localization of regional lymph node metastases. Eur J Nucl Med 25(11): 1495-501.

12. Erdi YE, Rosenzweig K, Erdi AK, Macapinlac HA, Hu YC, et al (Radiotherapy Treatment Planning for patients with non-small cell lung cancer using positron emission tomography (PET). Radiother Oncol 62: 51-60.

13. Jeffrey Bradley, Dehdashti F, Siegel BA, Bosch W, Bertrand RJ, et al. (2004) Impact of FDG-PET on radiation therapy volume delineation in non-small-cell lung cancer. Int J Radiation Oncology Biol Phys 59(1): 78-86.

14. Lawrence, Yorke, Andrew, Randall K Ten Haken, Avraham Eisbruch, et al. (2010) Use of Normal Tissue Complication Probability Models in the Clinic. Int J Radiation Oncology Biol Phys 76(3): S10-S19.

\section{Your next submission with Juniper Publishers will reach you the below assets}

